\title{
Abordaje subcraneal. Consideraciones técnicas y aplicaciones en patología trau- mática craneofacial
}

\author{
I. Zubillaga-Rodríguez; M.I. Falguera-Uceda; G. Sánchez-Aniceto; J.J. Montalvo-Moreno y R. Díez-Lobato*
}

Servicios de Cirugía Oral y Maxilofacial y de Neurocirugía Hospital Universitario 12 de Octubre. Madrid.

\section{Resumen}

Introducción. La elección del abordaje más adecuado a la base craneal en cada caso es esencial para la obtención de resultados quirúrgicos globales satisfactorios. Inicialmente dependerá de la localización anatómica exacta de la lesión dentro de la base craneal y de su extensión tridimensional, así como de la naturaleza de la misma. Las opciones posibles deben ser depuradas finalmente en función del status global del paciente, planificación reconstructiva y experiencia del equipo quirúrgico. El abordaje subcraneal es una opción terapéutica segura en el tratamiento de la patología traumática frontal. Permite el manejo adecuado del seno frontal y su obliteración si es precisa, favoreciendo el seguimiento radiológico del mismo.

Objetivos. Validar el abordaje subcraneal como vía adecuada de acceso en patología traumática de la base craneal anterior. Revisión de nuestra serie clínica. Valoración de aspectos técnicos quirúrgicos. Análisis de complicaciones.

Material y métodos. Estudio retrospectivo de 50 pacientes intervenidos desde Enero de 2004 hasta Diciembre 2009 por el Servicio de Cirugía Maxilofacial y Servicio de Neurocirugía del Hospital Universitario 12 de Octubre (Madrid) mediante abordaje subcraneal. 34 pacientes presentaban traumatismos craneofaciales o secuelas postraumáticas y 16 pacientes presentaban patología oncológica. Se enumeran los casos oncológicos como base de experiencia para la descripción de los aspectos técnicos. La casuística descrita se refiere a la patología traumática y sus secuelas. Los principales parámetros analizados fueron la técnica quirúrgica incluyendo el material de relleno para la obliteración del seno frontal, patología traumática asociada, estancia hospitalaria y tasa de complicaciones.

Resultados. No existió mortalidad intra-postoperatoria inmediata. La edad varía entre los 15-76 años siendo 22 varones y 12 mujeres. Se describe

Recibido: 25-01-10. Aceptado: 2-08-10 el tipo de fracturas frontales asociadas. El material de relleno empleado en la obliteración frontal fue hueso de calota. El $29 \%$ de los pacientes con patología traumática o secuelas presentaron morbilidad precoz-tardía. La estancia media hospitalaria fue de 13 días.

Conclusiones. El abordaje subcraneal a la base craneal anterior es una técnica versátil y razonable en el tratamiento quirúrgico de la patología de dicha región anatómica. Aporta una exposición excelente de la cavidad nasal, órbitas y celdillas etmoidales-seno esfenoidal, permitiendo un amplio acceso a la fosa anterior con mínima retracción de los lóbulos frontales.

PALABRAS CLAVE: Abordaje subcraneal. Traumatismos craneofaciales. Viruta ósea de calota. Morbilidad.

Subcranial approach. Technical aspects and application in craneofacial traumatic pathology

Summary

Introduction. Suitable approach to anterior cranial base is mandatory to get global satisfactory surgical outcomes. In the beginning it depends on the exactly anatomical position into the cranial fossa and tridimensional spread. Surgical approach implies the evaluation of the patient status, reconstructive options and surgical team experience. Subcranial approach is a safe surgical option in the treatment of frontal traumatic pathology. It allows adequate management of frontal sinus and its obliteration with easy radiologic follow-up.

Objectives. To analyse subcranial approach as a treatment option in traumatic pathology of the anterior cranial base and to present our review of subcranial approach. Valuation of surgical technical aspects. and related complications.

Material and methods. Retrospective analysis of 50 patients operated (subcranial approach) from January 2004 to December 2009 by Maxillofacial and Neurosur- 
gery Department, University Hospital 12 de Octubre, Madrid. 34 patients presented craniofacial trauma or postraumatic sequela and 16 patients presented craniofacial tumours. Oncological cases offers experience to discuss surgical aspects. Results are related to traumatic pathology and sequela. Main items review were surgical technique including materials used for frontal sinus obliteration, associated traumatic pathology, hospital stay and complication rates.

Results. No perioperatory mortality was found. Patients' age ranged 15-76 years. 22 were male and 12 female. Description of frontal fractures involved. Frontal sinus obliteration was made with calvarian bone dust. Morbidity rates was $29 \%$ in posttraumatic patients. Mean hospital stay was 13 days.

Conclusions. Subcranial approach to anterior cranial base is a safe and reliable treatment option to the pathology of this area. It allows outstanding exposure of the nasal cavity, orbits, ethmoidal cells-sphenoid sinus and great access to anterior fossa without frontal lobe retraction.

KEY WORDS: Subcranial approach. Craniofacial trauma. Calvarian bone dust. Morbility.

\section{Introducción}

La elección del abordaje más adecuado a la base craneal en cada caso es esencial para la obtención de resultados quirúrgicos globales satisfactorios. Inicialmente dependerá de la localización anatómica exacta de la lesión dentro de la base craneal y de su extensión tridimensional, así como de la naturaleza de la misma. Las opciones posibles deben ser depuradas finalmente en función del status global del paciente, planificación reconstructiva y experiencia del equipo quirúrgico.

El seno frontal se sitúa en la unión entre el esplacnocráneo y el neurocráneo, entre la fosa craneal anterior y la región nasoetmoidoorbitaria. El tratamiento inapropiado del complejo frontal puede suponer serias complicaciones, principalmente infecciosas, incluso varios años después del suceso. Los pacientes con patología que afecte al seno frontal requieren un seguimiento a largo plazo incluyendo anualmente la realización de una prueba de imagen de control. De esta forma es posible el diagnóstico de complicaciones previas a su presentación clínica.

El abordaje subcraneal representa un paso fundamental en el desarrollo de las técnicas quirúrgicas de la base craneal. Es un avance lógico en el progreso de las osteotomías disponibles, fruto del esfuerzo y cooperación simultánea entre las distintas especialidades que tratan la base del cráneo. Permite una amplia y óptima exposición de todos los planos de la fosa craneal anterior, desde el

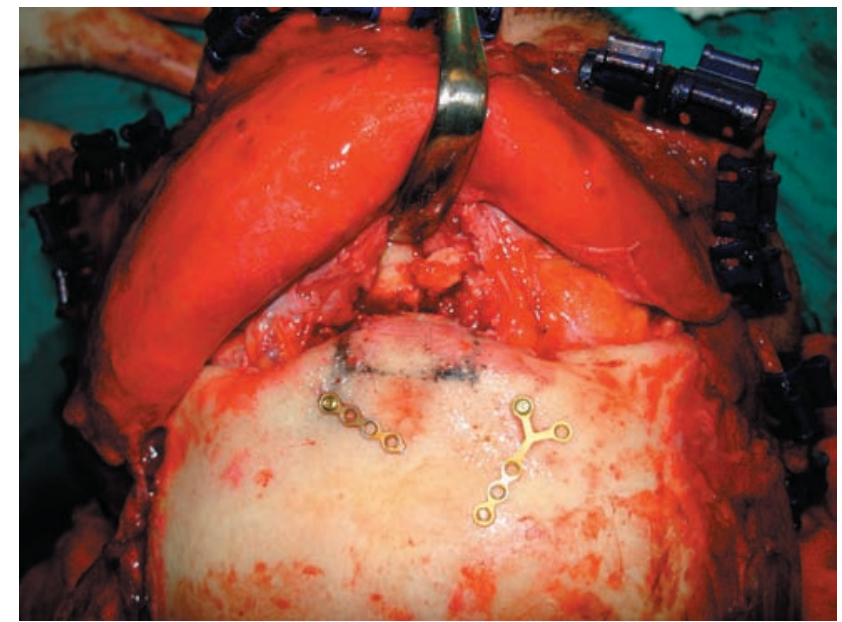

Figura 1. Diseño de osteotomía de abordaje.

techo etmoidal anterior hasta el clivus-planum esfenoidale incluyendo los techos orbitarios. Este objetivo se logra sin retracción de los lóbulos frontales evitando de esta manera la morbimortalidad asociada que dicha maniobra conlleva. Favorece el tratamiento de fracturas y sus secuelas tras impactos de alta energía con afectación de la fosa craneal anterior, incluso con desgarros durales y herniación del tejido cerebral, la descompresión del nervio óptico, la corrección de malformaciones craneofaciales y el aislamiento estrictoreconstrucción de la base craneal anterior ${ }^{8}$.

\section{Material y métodos}

El abordaje subcraneal fue realizado en 50 pacientes en esta serie clínica en el Hospital Universitario 12 de Octubre, siendo realizado en todos los casos por el mismo equipo quirúrgico perteneciente a los servicios de Cirugía Maxilofacial y Neurocirugía.

Respecto a la etiología de la patología de base a tratar, 24 pacientes presentaban traumatismos craneoencefálicoscraneofaciales agudos, 16 con patología oncológica y 10 mostraban secuelas postraumáticas de la base craneal anterior. Se enumeran los casos oncológicos como base de experiencia para la descripción de los aspectos específicos de la técnica quirúrgica. Los resultados analizados se refieren a la casuística traumática, incluyendo patología traumática aguda y secuelas postraumáticas.

Respecto a la técnica quirúrgica, la incisión bicoronal clásica es modificada realizándola en zig-zag, iniciando la misma próxima a la raíz del hélix y con dirección posterior, asegurando la preservación de los vasos temporales superficiales, útiles no sólo como pedículo nutriente de colgajos locales empleados en la reconstrucción craneal sino también como vasos receptores si fuera necesario emplear técnicas microquirúrgicas reconstructivas.

En el momento actual, las técnicas de imagen tipo TAC 


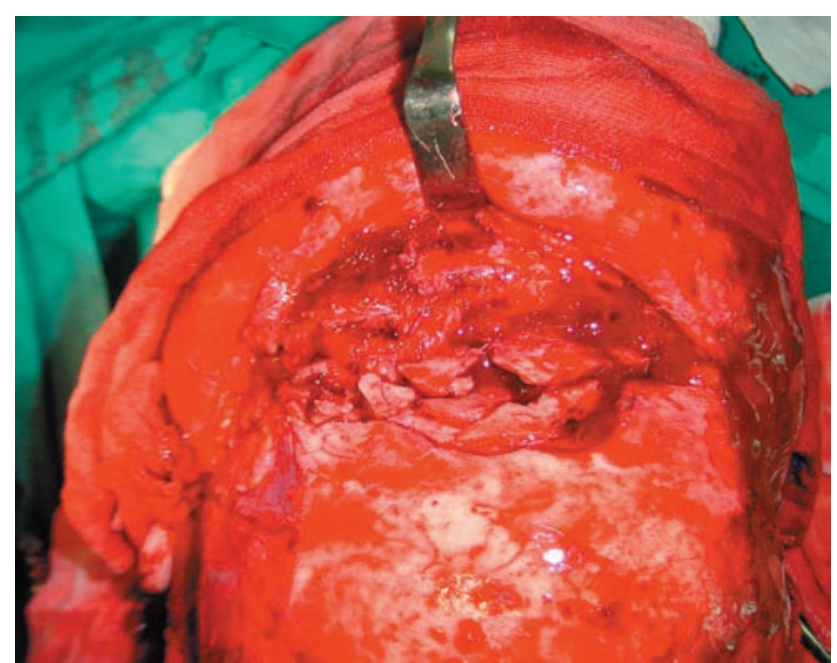

Figura 2. Fractura conminuta de pared anterior de seno frontal.

craneofacial permiten la evaluación de la forma y tamaño sinusal facilitando así la posterior osteotomía cuadrangular de acceso en la pared anterior del seno frontal. (Fig.1).

En traumatismos agudos, la osteotomía de abordaje al seno frontal es modificada siguiendo el patrón de fractura de la pared anterior del seno frontal (Fig. 2). La extracción de fragmentos óseos fracturados de la pared anterior permite el acceso al seno. Dichos fragmentos son conservados en suero salino para su posterior reposición anatómica tras el tratamiento adecuado del seno frontal. Es imprescindible una planificación adecuada del colgajo óseo teniendo en cuenta las fracturas existentes, siendo el objetivo final una exposición y manejo preciso de la patología traumática frontal.

En ausencia de traumatismos agudos, es preciso realizar la osteotomía de abordaje al seno frontal. Como el tamaño del colgajo óseo es conocido antes de realizar la osteotomía completa, es posible en este momento la preinserción de las miniplacas que fijarán la osteotomía en su posición definitiva ${ }^{28}$. Dichas miniplacas son retiradas parcialmente (se liberan los tornillos que la fijan al colgajo óseo y se rotan), se completa la osteotomía de la pared anterior y se aborda el seno. La osteotomía es realizada con sierra sagital con una angulación aproximada de $30^{\circ}$. Dicha sierra permite crear la osteotomía superior del colgajo de forma biselada como medida de seguridad para evitar penetrar en el sistema nervioso central a través de la fosa craneal anterior. Un osteotomo tras la glabela permite la separación del colgajo de la crista galli, evitando penetrar la dura frontal. La precisa colocación final del colgajo óseo asegura una adecuada reconstrucción frontal. El colgajo óseo es preservado en suero salino hasta su reposición final.

El colgajo óseo puede incluir los huesos propios nasales (Fig. 3). La osteotomía nasofrontal puede ser realizada por

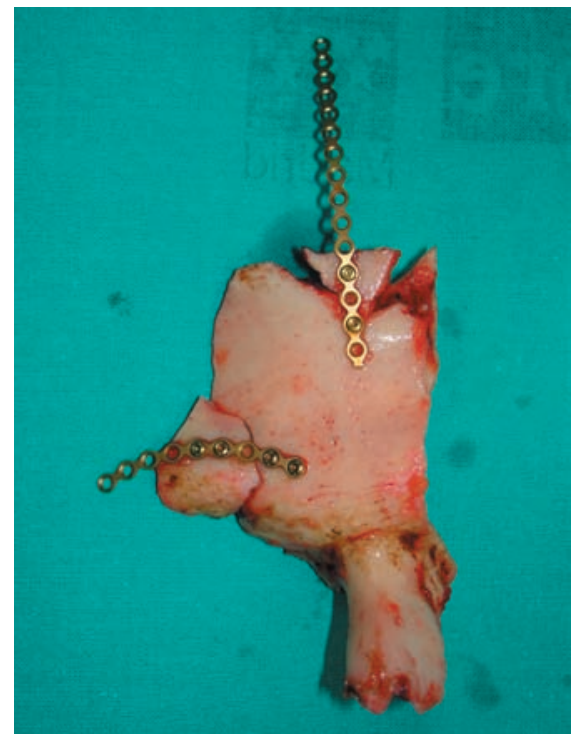

Figura 3. Osteotomía frontonasal con miniplacas preformadas.

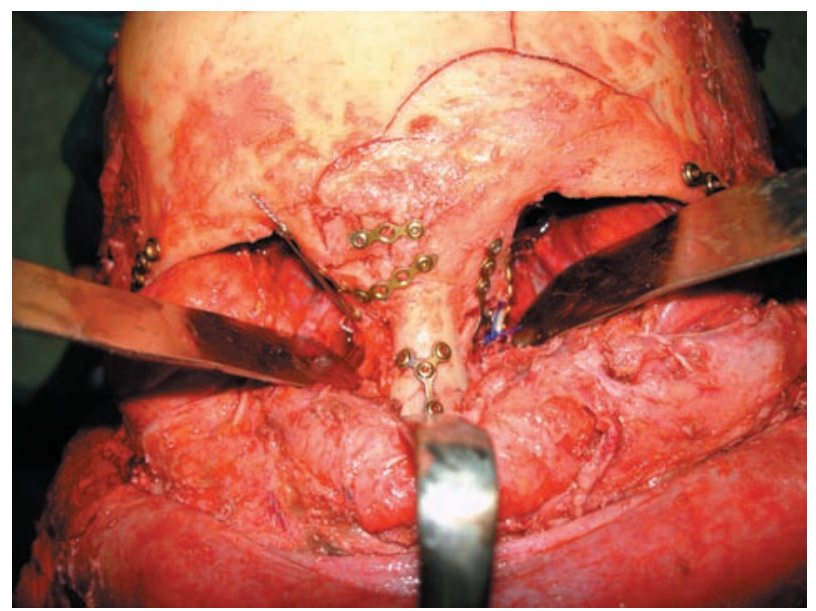

Figura 4. Cantopexia transnasal,

encima de las inserciones del ligamento cantal medial ${ }^{4}$. En estos casos es precisa la fijación de los ligamentos cantales mediante sutura no reabsorbible en la pared anterior del seno frontal contralateral pasando tras el segmento nasofrontal (cantopexia transnasal) (Fig. 4). Se obtiene así una correcta posición de los cantos mediales en los planos vertical, horizontal y sagital. Algunos autores preservan 3$5 \mathrm{~mm}$ distales de los huesos propios nasales intactos para mantener la funcionalidad de las válvulas nasales internas.

Si la patología de base afecta a un sólo seno frontal y el septum intersinusal no se encuentra afectado no existe indicación para la eliminación de dicho septum. Queda claro que requerirá tratamiento quirúrgico sólo el seno frontal afectado siempre y cuando el septum esté intacto.

Tras abordar el seno frontal se procede al tratamiento del proceso patológico implicado. Si existen fragmentos 


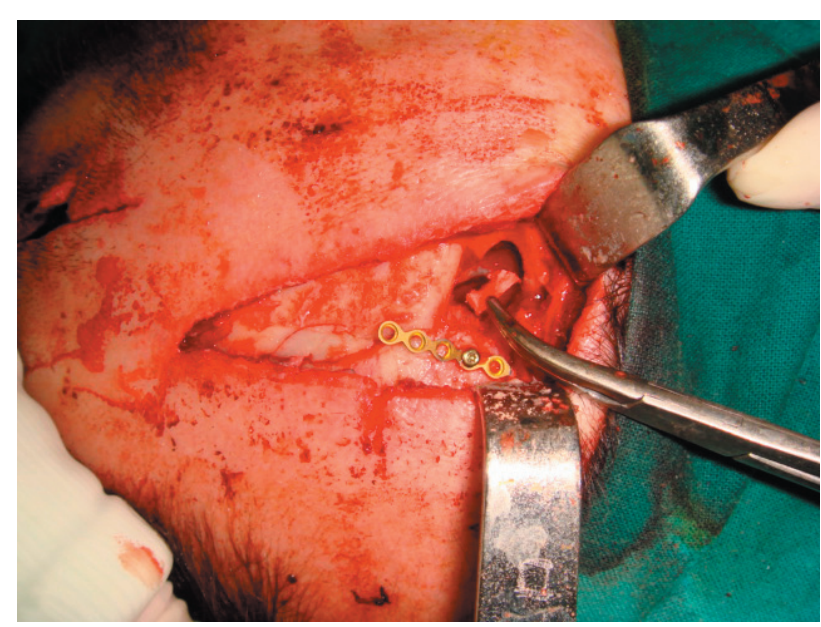

Figura 5. Obturación de ducto nasofrontal con hueso de calota.

óseos conminutos en la pared posterior son extraídos tras meticulosa disección de la duramadre para permitir la retracción de la porción anteroinferior de los lóbulos frontales y delimitar la extensión de la lesión a lo largo de la placa cribiforme y los techos orbitarios.

La mucosa del seno frontal es meticulosamente eliminada de las paredes sinusales ${ }^{14}$, procediendo posteriormente al fresado de las mismas. De esta forma, conseguimos eliminar las invaginaciones óseas de la mucosa sinusal disminuyendo las complicaciones postoperatorias. El fresado de las paredes óseas incrementa también el aporte vascular útil para el material de relleno empleado en la obturación del seno frontal.

El conducto nasofrontal ${ }^{7}$ es obturado con diversos materiales previa eliminación de la mucosa del mismo. El abordaje coronal realizado para el manejo de dichas patologías favorece la obtención de hueso autólogo como material adecuado para la obturación del conducto. (Fig. 5).

La obliteración del seno frontal se define como la ocupación de la cavidad aérea sinusal frontal manteniendo intactas las paredes óseas. Se emplea en el manejo de patología traumática o de sus secuelas y patología oncológica. $\mathrm{Su}$ objetivo es la eliminación sinusal como unidad funcional, evitando la regeneración epitelial sinusal.

La obliteración sinusal fue realizada con viruta ósea de calota (Fig. 6-8), favorecido dicho procedimiento por el abordaje coronal ${ }^{9}$. La consolidación ósea sinusal frontal que se obtiene con este método permite a largo plazo un exhaustivo control radiológico, siendo más sencillo el diagnóstico de posibles recidivas de la patología de base (Fig. 9). Tras la obliteración sinusal se recoloca el colgajo óseo frontal y se fija en su posición predeterminada inicialmente (Fig. 10). La osteosíntesis habitualmente es realizada con miniplacas de titanio (Fig. 11) (1.3 mm) aunque es posible

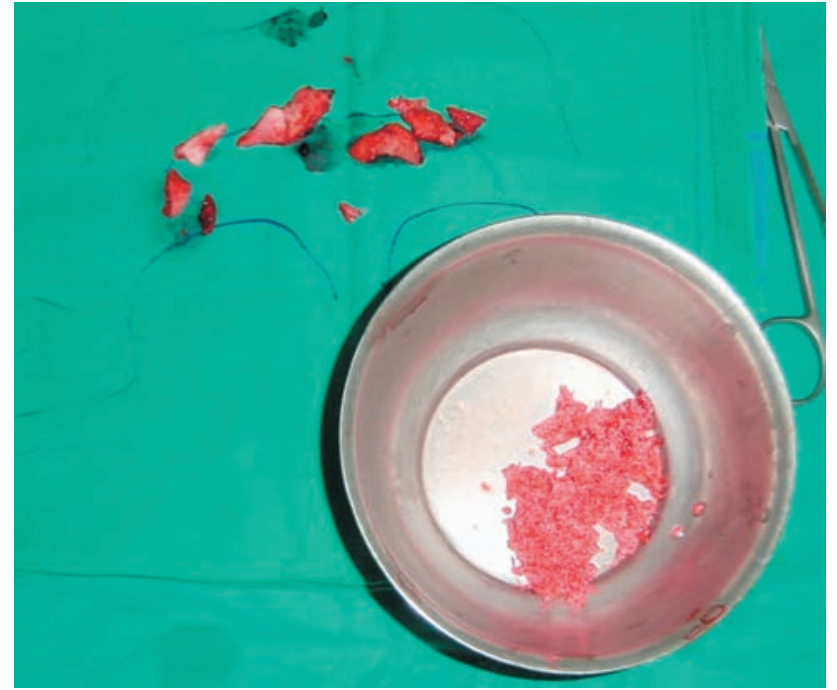

Figura 6. Calota obtenida para obliteración sinusal.

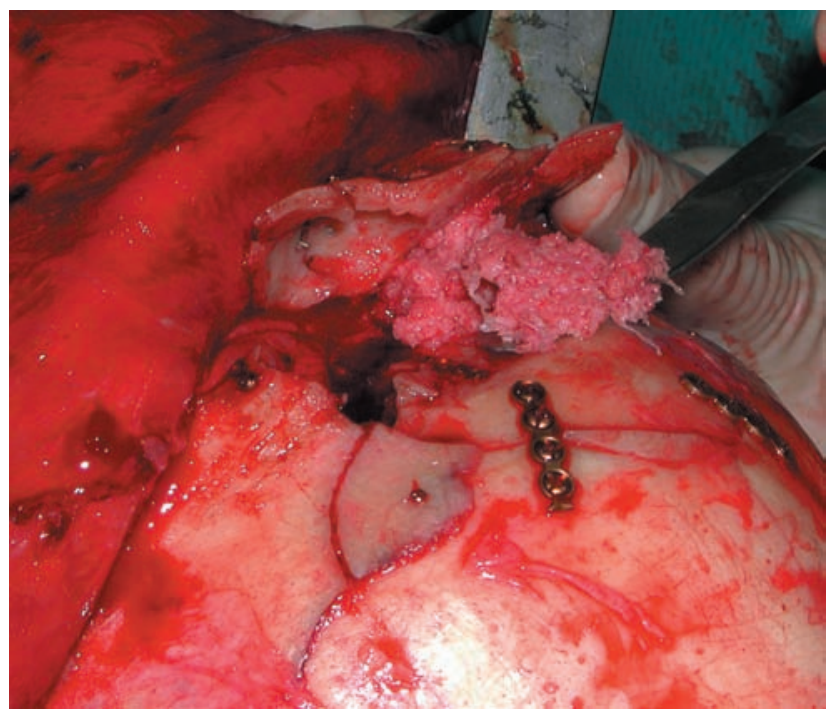

Figura 7. Obliteración sinusal con virutas óseas de calota.

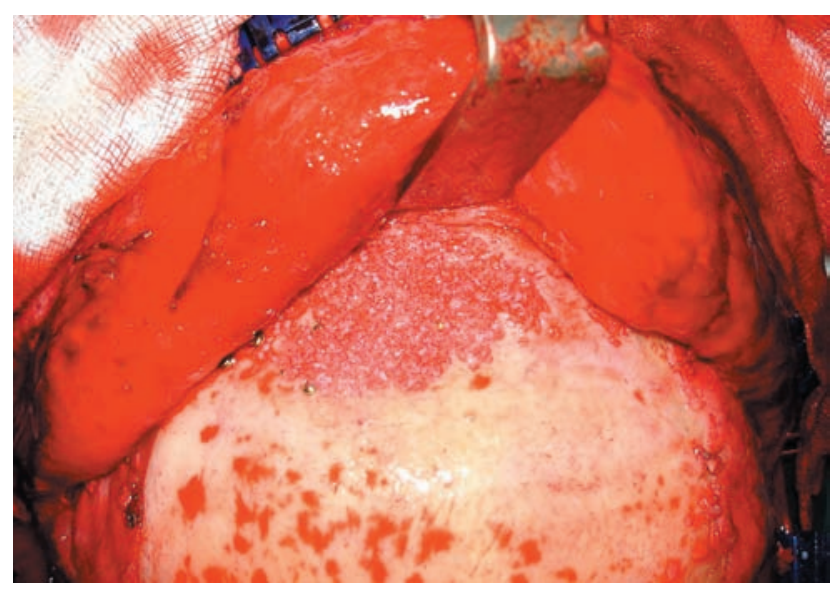

Figura 8. Aspecto final tras la obliteración completa. 


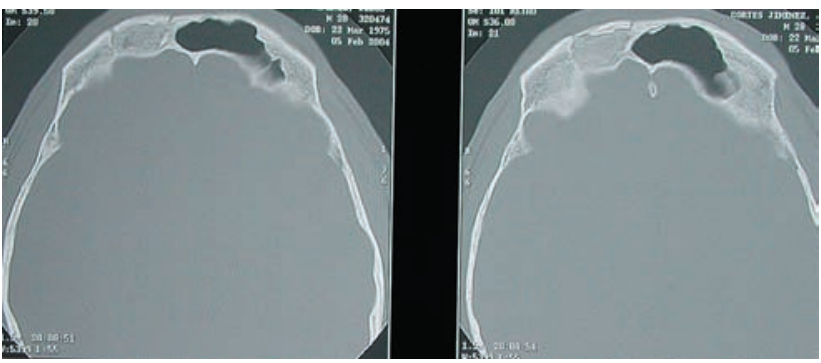

Figura 9. TAC axial donde se objetiva la consolidación ósea sinusal.

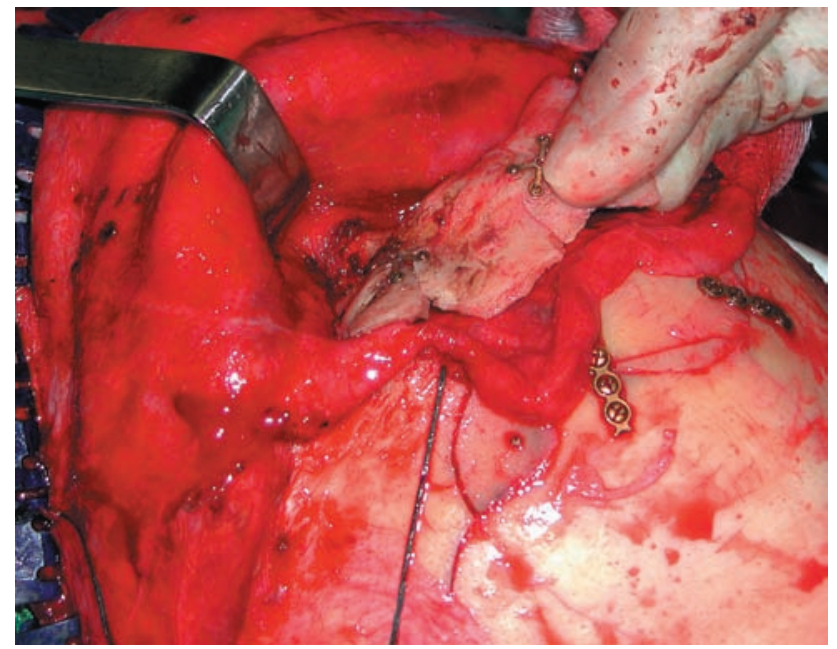

Figura 10. Fijación definitiva de colgajo óseo frontal.

el uso de osteosíntesis reabsorbible.

\section{Resultados}

Fueron realizados un total de 34 abordajes subcraneales en 34 pacientes, 22 varones y 12 mujeres.

El rango de edad osciló entre 15 y 76 años, siendo la edad media de 36 años. El período de seguimiento varía desde los 12-72 meses, siendo el seguimiento medio de 48 meses.

La edad media de los pacientes con patología traumática aguda fue de 33 años y la de los pacientes con secuelas de traumatismos de la base craneal anterior fue de 41 años.

Desde el punto de vista de la etiopatogenia traumática, el mecanismo de acción habitual es el impacto directo de alta energía en la región frontal tras accidente de tráfico $(58 \%)$, precipitación tras accidente laboral $(27 \%)$, agresiones $(13 \%)$ y accidentes deportivos $(2 \%)$.

Globalmente, 24 pacientes presentaban traumatismos craneoencefálicos-craneofaciales agudos. En un $63 \%$ de los casos se trataba de pacientes politraumatizados.

$60 \%$ de los pacientes presentaban fracturas frontales abiertas con heridas asociadas supraciliares-scalp frontal. La localización-dimensión de las heridas asociadas permi-

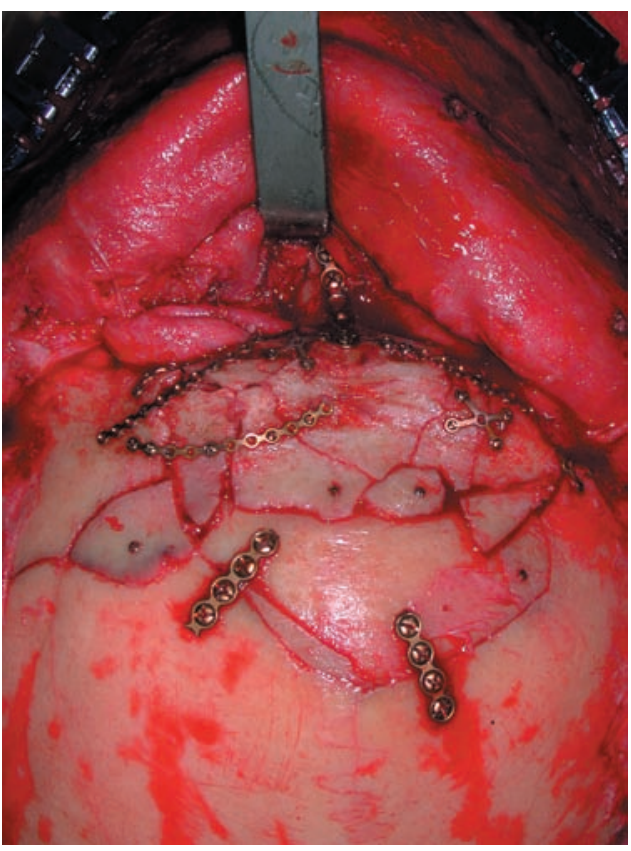

Figura 11. Osteosintesis habitual con miniplacas de titanio.

tió el tratamiento a través de las mismas en 3 pacientes. En el resto fue preciso recurrir al abordaje coronal.

Clasificamos las fracturas del seno/s frontales según su localización, grado de desplazamiento y presencia de conminución. Así, el patrón de fractura más frecuente fue el bilateral con desplazamiento de pared anterior y fractura lineal posterior (23.3\%) En orden decreciente se sitúan el patrón bilateral con conminución anterior y desplazamiento de pared posterior $(16.6 \%)$; unilateral con desplazamiento anterior-fractura lineal posterior $(13.3 \%)$; bilateral con desplazamiento anterior-posterior $(10 \%)$; bilateral con fractura lineal anterior-posterior (6.6\%); unilateral con desplazamiento anterior y posterior $(6.6 \%)$; unilateral con fractura lineal anterior y desplazamiento posterior $(6.6 \%)$; unilateral con desplazamiento anterior y conminución posterior (6.6\%); bilateral con conminución anterior y fractura lineal posterior (3.3\%); unilateral con conminución anterior y desplazamiento posterior (3.3\%) y unilateral con fractura lineal anterior-posterior (3.3\%).

En un 53.3\% de los casos el septum intersinusal frontal se hallaba fracturado y en el $90 \%$ se objetivó afectación intraoperatoria del ducto nasofrontal.

Respecto a las fracturas faciales asociadas (Tabla I), un $76.6 \%$ se encontraban en el contexto de fracturas panfaciales. Las fracturas más frecuentemente asociadas fueron las del reborde supraorbitario (uni o bilateral, $86.6 \%$ ) y de la pared medial orbitaria (uni o bilateral, $80 \%$ ).

$73.3 \%$ asociaban fracturas del esqueleto óseo nasal y $56.6 \%$ fractura del suelo orbitario $(52 \%$ con desplazamiento y $48 \%$ sin desplazamiento). $53.3 \%$ presentaban 


\section{Tabla I}

Fracturas faciales asociadas a fracturas frontobasales

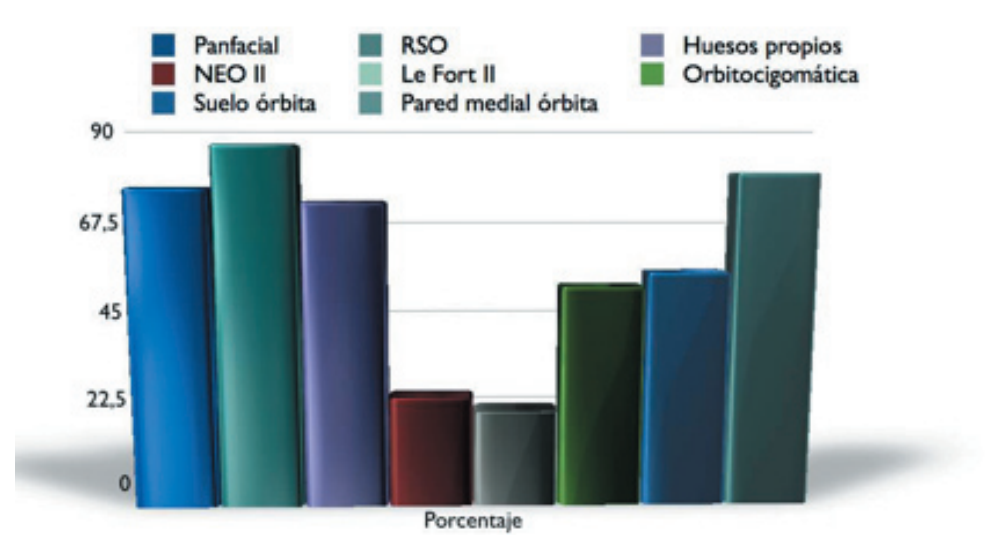

RSO: reborde supraorbitario. NEO II: nasoetmoidoorbitaria tipo II.

fracturas orbitocigomáticas (63\% de media energía, 33.3\% de alta energía y $3.7 \%$ de baja energía). En relación con fracturas tipo Lefort, el subtipo más frecuente fue el II (23.3\%), seguido del I y III (ambos 6.6\%). La fractura nasoetmoidoorbitaria (NEO) más frecuente fue la tipo II unilateral $^{18}$, en un $26.6 \%$. 3 pacientes presentaron fractura de mandíbula ( 2 dobles, parasinfisaria-cuerpo ipsilateral y parasinfisaria-subcondílea contralateral y 1 simple, parasinfisaria) en el contexto de fracturas panfaciales.

En los casos con patología traumática aguda de la base craneal anterior también se analizaron los signos neuroquirúrgicos-oftalmológicos asociados.

Al ingreso hospitalario (Tabla II), el traumatismo craneoencefálico fue leve en el $56.6 \%$ de los casos, moderado en el $30 \%$ y grave en el $13.4 \%$.
En el TAC cerebral inicial se objetivaba neumoencéfalo $(70 \%)$, contusión frontal $(53.3 \%)$ y hematoma epidural (43.3\%). 7 pacientes presentaban fístula de líquido cefalorraquídeo activa en la exploración clínica inicial; 3 presentaron pérdida de masa encefálica frontal ("fractura frontal through and through") y un paciente debutó con un absceso intraparenquimatoso que requirió su drenaje quirúrgico previo a la intervención definitiva subcraneal.

Respecto a las lesiones oftalmológicas, todos los pacientes presentaban equimosis conjuntival $(56.7 \%$ bilateral y $43.3 \%$ unilateral). Se apreció neuropatía óptica postraumática en 9 pacientes, 3 de ellos con sección del nervio óptico. Otros signos asociados son el edema retiniano $(16.7 \%)$, perforación ocular $(10 \%)$ y uveitis anterior, desprendimiento de retina y luxación de cristalino $(\mathrm{n}=1)$.

Tabla II

Signos neuroquirúrgicos en fracturas frontobasales al ingreso

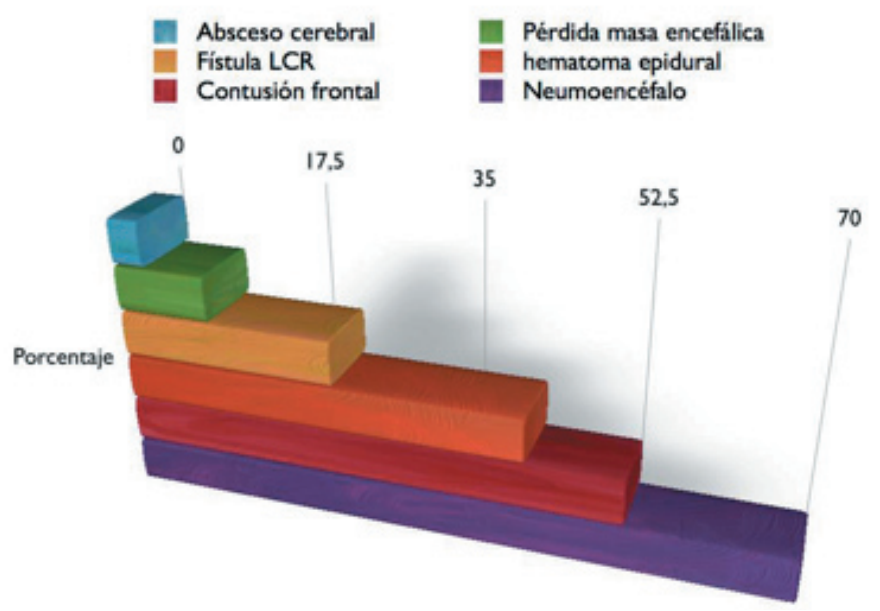

LCR: Líquido cefalorraquídeo 


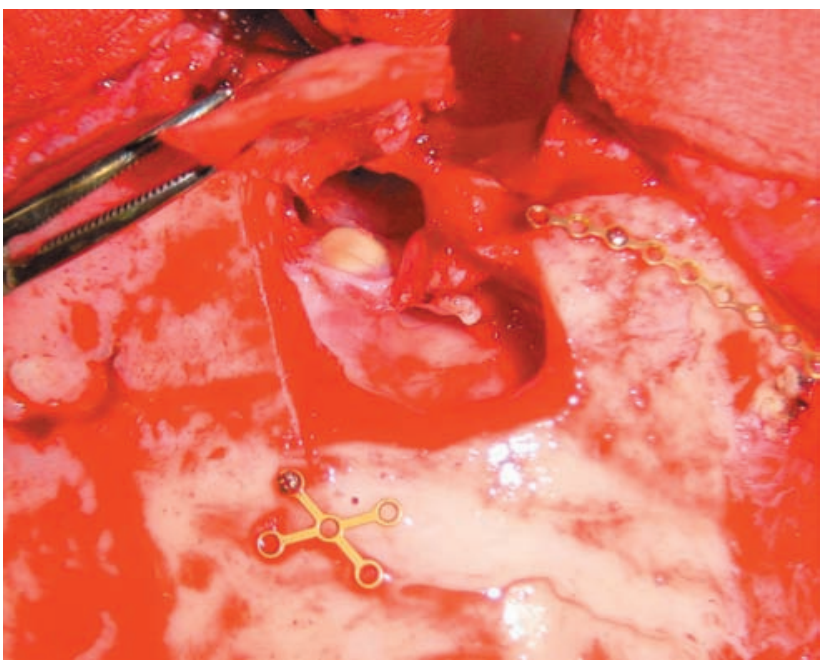

Figura 12. Defecto óseo en pared posterior, afectación dural con fistula de líquido asociada.

En el contexto de pacientes politraumatizados, la patología traumática que más se asoció fue el trauma cervical, seguido de las contusiones costales.

Las secuelas postraumáticas de la base craneal anterior se dividen en infecciosas (mucopioceles postraumáticos $\mathrm{n}=7$ ) y fístulas de líquido cefalorraquídeo en pacientes con fracturas de la base craneal anterior no tratados quirúrgicamente en el momento inicial ( $\mathrm{n}=3$ ) (Fig. 12). El tiempo medio de desarrollo de las secuelas infecciosas postraumáticas fue de 19 meses (18.85) y de las fístulas de líquido fue de 9 meses.

La estancia media hospitalaria global fue de 13 días. La estancia media en pacientes con patología traumática fue de 16 días y en secuelas de traumatismos craneofaciales fue de 9 días.

No existió mortalidad perioperatoria. No se produjo necrosis parcial-total de ningún colgajo óseo frontal. La obliteración del seno frontal con viruta ósea de calota permite un adecuado seguimiento radiológico a largo plazo, favoreciendo el diagnóstico precoz de las posibles complicaciones sinusales asociadas.

10 pacientes con patología traumática o secuelas (29\%) presentaron complicaciones (Tabla III) tras la intervención quirúrgica (50\% precoces, antes de los 6 meses postcirugía y $50 \%$ tardías). El $60 \%$ de los pacientes con complicaciones precisaron reintervención quirúrgica (drenaje de hematoma epicraneal, correción de enoftalmos postraumático con injertos de costilla $+/$ - mallas de titanio $(n=2)$, drenaje de absceso maseterino, intervención de Caldwell-Luc en sinusitis maxilar y craneoplastia para correción de deformidad estética frontal). 2 pacientes desarrollaron celulitis frontoorbitarias que respondieron a antibioterapiaantiinflamatorios i.v. y un paciente presentó fístula de líquido cefalorraquídeo persistente en el postoperatorio inmediato que cedió con reposo sin necesidad de drenaje lumbar. El paciente con dolor crónico frontal fue tratado con analgesia pautada.

\section{Discusión}

Podemos establecer unos principios básicos o reglas de oro para la elección del abordaje más adecuado a la base craneal:

1. Elegir la vía de acceso más próxima hasta nues-

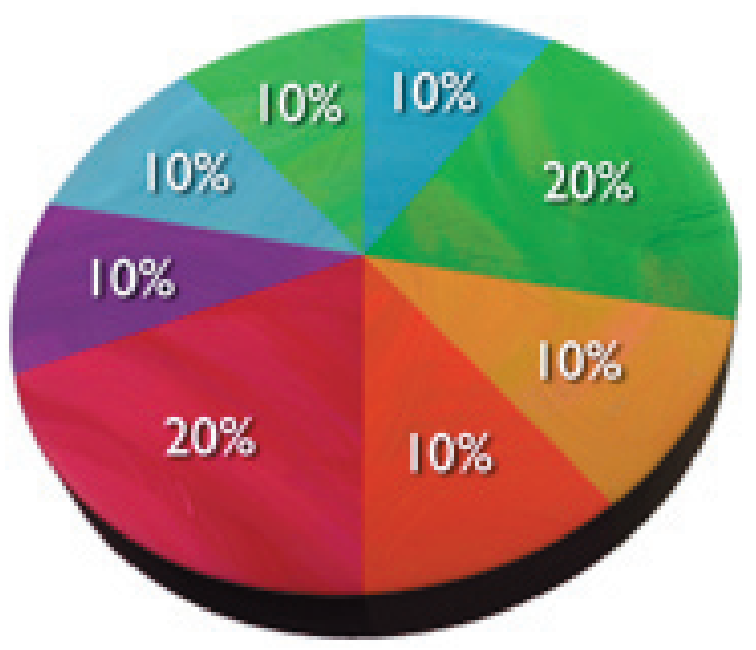


tro objetivo, sorteando y protegiendo aquellas estructuras nobles que aseguren una supervivencia funcionalmente aceptable del paciente.

2. Considerar siempre posibles alteraciones de la vascularización si han existido cirugías previas y/o radioterapia.

3. Realizar las osteotomías craneofaciales necesarias para evitar una excesiva retracción cerebral y conseguir una adecuada exposición del objetivo que permita el manejo más satisfactorio de la lesión.

4. Las osteotomías deben procurar fragmentos óseos únicos y se deben practicar con las osteosíntesis preformadas para asegurar una correcta reposición anatómica.

5. Elección del abordaje en estrecha relación con la planificación reconstructiva, especialmente para obtener un adecuado aislamiento del sistema nervioso central de las vías aerodigestivas superiores.

6. Lograr el mejor resultado estético posible.

7. Minimizar al máximo la morbimortalidad del paciente.

Aunque los métodos tradicionales de abordaje de lesiones frontobasales complejas están firmemente establecidos, son seguros y efectivos, presentan altas tasas de morbilidad fundamentalmente por retracción de los lóbulos frontales (edema, contusión, encefalomalacia). Dicha morbilidad se incrementa especialmente si existe afectación del planum esfenoidale o de la región selar. Además, las incisiones cutáneas faciales realizadas en los abordajes clásicos transfaciales presentan el inconveniente de las cicatrices faciales y la morbilidad estética asociada ${ }^{11}$.

Las siguientes desventajas están relacionadas con los abordajes intracraneales:

1. Alta tasa de morbilidad, incluyendo anosmia, edema postoperatorio, estancia hospitalaria prolongada $\mathrm{y}$ riesgo elevado de infección aguda-crónica de SNC.

2. La demora en el tratamiento reconstructivo definitivo (2-3 semanas) complica dicho procedimiento (formación de tejido de granulación, incremento en la tasa de infección postoperatoria, drenaje inadecuado de las cavidades sinusales) y empeora su resultado.

Estas desventajas dieron lugar al desarrollo de un nuevo abordaje que permitiera el manejo adecuado completo de la fosa craneal anterior como alternativa a los tradicionales abordajes intracraneales.

El abordaje subcraneal representa un acceso extracraneal y fue descrito inicialmente por Joram Raveh en 1978 para el tratamiento de los traumatismos complejos de la base craneal anterior ${ }^{25,26}$. Se cimenta en estudios pioneros de Frazier, Smith y Ketcham ${ }^{15}$ quienes demuestran las posibilidades de los abordajes transfaciales y tras los avances obtenidos por Tessier y Derome ${ }^{7}$ en relación al abordaje transbasal. Fue posteriormente utilizado para la corrección de malformaciones craneofaciales congénitas y en 1980 se describe por primera vez su uso en cirugía oncológica basicraneal ${ }^{24}$.

La diferencia respecto a los procedimientos tradicionales no radica tanto en la localización anatómica de la craneotomía frontal y las osteotomías nasoorbitarias como en la diferente exposición y dirección del abordaje. Permite una amplia exposición inferior de todos los planos de la base craneal anterior (desde el techo etmoidal anterior hasta el clivus atravesando ambos techos orbitarios y el plano esfenoidal) así como de la porción media y superior de la cavidad nasal y el aspecto medial del seno maxilar. En lesiones con extensión caudal hacia la porción inferior de la cavidad nasal, suelo-antrum del seno maxilar y/o proceso alveolar maxilar-paladar se puede combinar con un abordaje tipo degloving mediofacial en el mismo procedimiento ${ }^{8}$. Otras técnicas que pueden ser asociadas incluyen abordajes transfaciales como osteotomía tipo LeFort I y abordajes orbitocigomáticos-transorbitarios 9 .

Gran variedad de técnicas han sido empleadas para acceder al seno frontal ${ }^{1,3,5,30}$, como la realización de colgajos óseos o la transiluminación sinusal para desarrollar injertos óseos libres de la tabla anterior del seno frontal. La progresión de la cirugía del seno frontal ha sido paralela al desarrollo del tratamiento de los traumatismos craneofaciales.

Los conceptos y principios actuales se basan en la intervención quirúrgica precoz buscando obtener resultados estéticos y funcionales estables. En el momento actual, las técnicas de imagen tipo TAC craneofacial permiten la evaluación de la forma y tamaño sinusal facilitando así la posterior osteotomía cuadrangular de acceso en la pared anterior del seno frontal.

Las bases anatómicas para la eliminación de la cortical ósea interna mediante fresado fueron descritas por Mosher y $\mathrm{Judd}^{23}$. Describen pequeñas depresiones en la cortical interna que permiten el paso a las venas diploicas de Breschet. Estas venas conectan la mucosa con las venas durales. Insiste así en la necesidad de eliminación de dichas depresiones como sitios potenciales de retención mucosa.

Hilding $^{13}$, en sus trabajos sobre seno frontal en perros, muestra que la incompleta eliminación de la membrana mucosa implica una obliteración sinusal incompleta con mantenimiento de una cavidad aérea y formación posterior de quistes mucosos. Sugiere que las islas mucosas traumatizadas retenidas presentan un crecimiento anormal con tendencia a la formación de quistes mucosos que pueden originar mucoceles. Otros autores como Montgomery ${ }^{22}$ defienden incluso el uso del microscopio intraperatorio como ayuda para una meticulosa exéresis de la mucosa sinusal.

La afectación del conducto nasofrontal es una de las causas más comunes de complicaciones intracraneales. Habitualmente no es posible su estricta evaluación sin su 
abordaje quirúrgico. La obturación del conducto se realiza en caso de afectación del mismo.

El conducto nasofrontal es obturado con diversos materiales. De la literatura se extrae que los mejores resultados a largo plazo se obtienen cuando la obturación se realiza con hueso autólogo ${ }^{29}$. En los traumatismos craneofaciales con fracturas conminutas del tercio superior facial es posible el empleo de fragmentos óseos de mínimo tamaño para la obturación del conducto. Otros autores utilizan fascia, músculo o grasa con resultados satisfactorios. Con independencia del método usado, la obliteración del ducto sella la cavidad sinusal, previene el ascenso de procesos infecciosos desde las vías aerodigestivas superiores y previene el nuevo crecimiento de mucosa desde el conducto con el potencial riesgo de formación de mucoceles.

De la revisión previa se establecen los principios básicos para la obtención de una cavidad sinusal frontal afuncional $^{27}$ :

1. Meticulosa exéresis de toda la mucosa sinusal frontal.

2. Eliminación de la cortical interna de las paredes sinusales

3. Oclusión permanente del conducto nasofrontal.

A los principios básicos descritos se une la obliteración sinusal frontal. Dicho procedimiento se ha convertido en uno de los puntos clave en el tratamiento de fracturas, infecciones crónicas y procesos oncológicos benignos del seno frontal. Disminuye el riesgo de aparición de complicaciones infecciosas (mucoceles, mucopioceles, osteomielitis, meningitis y abscesos cerebrales) dada la potencial degeneración quística de la mucosa sinusal afectada.

Una de las controversias en torno al manejo del seno frontal se ha centrado tradicionalmente en el material empleado para el relleno de la cavidad sinusal tras el tratamiento de la patología de base.

Existen en la literatura estudios experimentales y clínicos defendiendo el uso de un material determinado como relleno de la cavidad. Sin embargo, la mayoría de estos estudios son realizados sobre senos frontales sin patología asociada (modelos animales).

Se han descrito varios procedimientos básicos para la obliteración del seno frontal como el empleo de grasa autóloga basado en los trabajos de Goodale y Montgomery $^{11}$. La implantación de músculo ${ }^{20}$ y el relleno con hueso autólogo son otros procedimientos empleados. La osteoneogénesis espontánea fue revisada por MacBeth ${ }^{19}$ en los años 50 mientras que Bosley ${ }^{3}$ presentó evidencias radiográficas con obliteraciones sinusales con hueso nuevo en 93 de los 100 pacientes tratados con este método. Finalmente, algunos autores optan por el empleo de materiales aloplásticos como relleno.

El empleo de hueso autólogo en la obliteración del seno frontal se ha convertido en una técnica eficaz tras los estudios realizados durante las últimas décadas ${ }^{29,31}$. Sufre una rápida vascularización útil para el relleno del seno frontal. Se produce un proceso de "creeping substitution", actuando como soporte para la consolidación ósea ${ }^{27}$. La neoformación ósea es estimulada tanto por los elementos celulares aportados por el injerto particulado como por los precusores osteogénicos de la zona receptora. Su uso no se estandarizó en el pasado posiblemente por la morbilidad asociada creada en la zona donante. Sin embargo, el empleo de un material de relleno osteoconductor favorece la consolidación ósea, motivo por el cual para muchos autores el hueso autólogo es el material de elección para la obliteración de cavidades aéreas. En los estudios revisados en los que el seno frontal fue obliterado con hueso autólogo se objetivó relleno de la cavidad con hueso neoformado. Sólo Micke $^{21}$ et al. describieron un $86 \%$ de obliteración sinusal cuando el hueso autólogo fue el material de relleno. Es difícil evaluar el grado de nuevo hueso formado con radiografías sólo, pero los estudios experimentales en animales muestran la obliteración por hueso neoformado tras el empleo de hueso autólogo.

En el momento actual, la obtención de hueso de calota craneal presenta una mínima morbilidad residual en el área donante. La toma de los injertos para la obliteración del seno se realiza mediante un rascador de hueso bajo irrigación externa asociada. De esta manera se obtienen virutas de calota procedentes de la tabla externa craneal excelentes para un relleno eficaz de la cavidad sinusal frontal. Si es necesaria la reconstrucción asociada de alguna pared del seno frontal, el mismo abordaje coronal favorece la obtención de injertos de calota (también tallados de la cortical externa).

Décadas después de la primera obliteración sinusal frontal, el material óptimo permanece desconocido y es fuente de frecuentes debates en el ámbito de la cirugía reconstructiva.

La multitud de métodos empleados atiende al hecho de que todos ellos han presentado un grado moderado de éxito. La revisión extensa de la literatura en torno a la obliteración del seno frontal sugiere que el material empleado en el relleno de la cavidad sinusal no es tan importante como la técnica quirúrgica empleada para la preparación del seno previa a la implantación del material.

Por otra parte, en casos postraumáticos, los intentos de restablecimiento de la funcionalidad del seno frontal cuando existe afectación del ducto nasofrontal tienen una tasa de fracaso elevada próxima al $30 \%$. Dicho fracaso es debido a la consiguiente retracción cicatricial y estenosis ${ }^{2,17}$ a nivel del ducto nasofrontal. En cualquier caso, algunos autores, cuando la pared posterior del seno frontal está intacta prefieren restablecer inicialmente la permeabilidad del conducto nasofrontal y preservar la función sinusal ${ }^{10}$. Se 
procede a la colocación de un tubo de drenaje de adecuado calibre recreando el ducto. Se mantiene en dicha posición durante un periodo de tiempo variable (desde 1 semana hasta 6 meses) para prevenir la retracción mucosa y la oclusión ductal durante el proceso de cicatrización ${ }^{12}$. Aunque el tratamiento en cada paciente debe ser individualizado, algunos autores opinan que la función del seno frontal y su anatomía pueden ser preservadas en la mayoría de casos teniendo en cuenta el grado de desplazamiento, el status del ducto nasofrontal, la conminución de la pared posterior y la presencia de afectación neurológica significativa o de afectación dural. En nuestra opinión y tras el análisis de los resultados, tanto los pacientes con sinusitis frontales de repetición como los que desarrollaron mucoceles se habían tratado de forma menos exhaustiva en la primera cirugía. Tras el desarrollo de complicaciones se optó por la obliteración sinusal con viruta ósea de calota, evolucionando de forma favorable. Así mismo, en pacientes en los que la obliteración sinusal se realizó en el tratamiento inicial no se observaron complicaciones infecciosas.

Respecto a las contraindicaciones para la realización del abordaje subcraneal se encuentran los traumatismos de la base craneal anterior laterales al techo orbitario o lesiones que requieren acceso neuroquirúrgico al lóbulo frontal vía craneotomía standard. Los límites laterales del abordaje incluyen los nervios ópticos, senos cavernosos y caudalmente las porciones laterales e inferiores del maxilar superior. La afectación del seno cavernoso, arteria carótida interna, quiasma óptico y la invasión cerebral masiva implican la necesidad de asociar un abordaje intracraneal para la resolución adecuada de la patología de base ${ }^{16}$.

La mayor desventaja es la anosmia. En los traumatismos con fracturas conminutas extensas del área cribiforme, la lesión de las cintillas olfatorias es inevitable. Independientemente del abordaje, la exposición completa superior de la base craneal anterior requiere la elevación de las cintillas olfatorias de la lámina cribiforme con la consiguiente pérdida de olfación. La anosmia reduce la apreciación del sentido del gusto. Aunque la pérdida del olfato es con frecuencia necesaria para erradicar enfermedades que afectan a la base craneal anterior, las cintillas olfatorias contralaterales pueden ser preservadas en el tratamiento de patología selectiva unilateral ${ }^{6}$.

\section{Conclusiones}

El aspecto reconstructivo de la cirugía de la base craneal ha ido ganando interés hasta el momento actual. La reconstrucción es una fase indispensable de cualquier procedimiento quirúrgico complejo. Cuanto mayor es la afectación de estructuras anatómicas críticas durante la cirugía, mayor es la demanda en la integridad de la reconstrucción. Hoy día no sólo es concebible una resolución adecuada de la patología de la base craneal sino que es mandatoria la obtención de resultados estéticos y funcionales satisfactorios. Los conocimientos y técnicas actuales permiten y propugnan el tratamiento precoz, agresivo y definitivo de la patología de la base craneal en un solo tiempo quirúrgico.

El abordaje subcraneal es un método versátil y eficaz de acceso a la base craneal anterior. Entre sus ventajas destaca:

1. La posibilidad en la mayoría de los casos de la reconstrucción craneofacial precoz en un solo acto quirúrgico (primeras 24-48 horas) a pesar de la afectación grave oncomitante de la base craneal, la presencia de fragmentos craneales desplazados y la contusión-edema y/o herniación de tejido cerebral.

2. Disminución significativa de la morbilidad asociada evitando la retracción de los lóbulos frontales. Reduce el edema cerebral y las fístulas de líquido cefalorraquídeo recurrentes postoperatorias, asociando una excelente exposición de la cavidad nasal, órbitas, celdillas etmoidales y seno esfenoidal.

3. El tratamiento del pseudohipertelorismo (compresión centrípeta simétrica cantal para manejo del telecanto), la descompresión del nervio óptico (tras etmoidectomía radical) y la meticulosa reparación del tercio medio facial pueden ser realizadas en una intervención quirúrgica.

4. Sellado hermético de desgarros durales y manejo de la base craneal a través de los espacios paranasales. Permite un estricto aislamiento del sistema nervioso central con baja tasa de aparición de fístulas de líquido cefalorraquídeo e infecciones epidurales, disminuyendo así el tiempo de hospitalización.

5. Procedimiento estéticamente muy aceptable, evitando incisiones faciales; la única incisión es la bicoronal, preferiblemente camuflada tras la línea capilar.

\section{Bibliografía}

1. Anderson, C.M.: External operation on the frontal sinus: causes of failure. Arch Otolaryngol 1932; 15: 739-745.

2. Baron, S.H., Dedo, H.H., Henry, C.R.: The mucoperiosteal flap in frontal sinus surgery (The Sewall-Boyden-McNaught operation) Laryngoscope 1973; 83: 1266-1280.

3. Bosley, W.R.: Osteoplastic obliteration of the frontal sinuses: a review of 100 patients. Laryngoscope 1972; 82: 1463-1476.

4. Burstein, F., Cohen, S., Hudgins, R., Boydston, W.: Frontal basilar trauma: classification and treatment. Plast Reconst Surg 1997; 99: 1314-1323.

5. Casiano, R.R., Cooper, J.: Anterior table free bone graft technique for frontal sinus obliteration. Otolaryngol Head Neck Surg 1992; 106: 363-366.

6. Dale, J., Browne, J.D., Mims, J.W.: Preservation of olfaction in anterior skull base surgery. Laryngoscope 2000; 
110: 1317-1322.

7. Derome, P.: Les tumours sphenoethmoidales. Possibilities d'exerese et de reparation chirurgicales. Neurochirurgie 1972; 18: 1-164.

8. Fliss, D.M., Zucker, G., Amir, A., Gatot, A., Cohen, J.T., Spektor, S.: The subcranial approach for anterior skull base tumors. Oper Tech in Otolaryngol Head neck Surg 2000; 11: 238-253.

9. Fliss, D.M., Abergel, A., Cavel, O., Margalit, N., Gil, Z.: Combined subcranial approaches for excision of complex anterior skull base tumors. Arch Otolaryngol Head Neck Surg 2007; 133: 888-896.

10. Gerbino, G., Roccia, F., Benech, A., Caldarelli, C.: Analysis of 158 frontal sinus fractures: current surgical management and complications. J Craniomaxillofac Surg 2000; 28: 133-139.

11. Goodale, R.L., Montgomery, W.W.: Experiences with the osteoplastic anterior wall approach to the frontal sinus; cases histories and recommendations. Arch Otolaryngol 1958; 68: 271-283.

12. Haug, R.H., Likavec, M.J.: Frontal sinus reconstruction. Oral Maxillofac Surg Clin North Am 1994; 2:,65-83.

13. Hilding, A.: Experimental surgery of the nose and sinuses: III. Results following partial and complete removal of the lining mucous membrane from the frontal sinus of the dog. Arch Otolaryngol 1933; 17:,760-768.

14. Kalavrezos, N.: Current trends in the management of frontal sinus fractures. Injury 2004; 35:,340-346.

15. Ketcham, A.S., Wilkins, R.H., VanBuren, J.M., Smith, R.R.: A combined intracranial facial approach to the paranasal sinuses. Am J Surg 1963; 106: 698-703.

16. Kinnunen, I., Aitasalo, K.: A review of 59 consecutive patients with lesions of the anterior cranial base operated on using the subcranial approach. J Craniomaxillofac Surg 2006; 34: 405-411.

17. Lindorf, H.H.: A contribution on revisional and drainage of the frontal sinus by osteoplastic operation. J Maxillofac Surg 1986; 14: 34-39.

18. Markowitz, B.L., Manson, P.N., Sargent, L., et al.: Management of the medial canthal tendon in nasoethmoid orbital fractures: the importance of the central fragment in classification and treatment. Plast Reconstr Surg 1991; 87: 843-853.

19. MacBeth, R.: The osteoplastic operation for chronic infection of the frontal sinus. J Laryngol Otol 1954; 68: 465-477.

20. Mcneil, R.A.: Surgical obliteration of the maxillary sinus: a clinical and experimental study. Laryngoscope 1967; 77: 202-217.
21. Mickel, T.J., Rohrich, R.J., Robinson, Jr J.B.: Frontal sinus obliteration: a comparison of fat, muscle, bone and spontaneous osteogenesis in the cat model. Plast Reconstr Surg 1995; 95: 586-592.

22. Montgomery, W.W., Pierce D.L.: Anterior osteoplastic fat obliteration for frontal sinus: Clinical experience and animal studies. Trans Am Acad Ophthalmol Otolaryngol 1963; 67: 46-57.

23. Mosher, H.P., Judd, D.K.: An analysis of seven cases of osteomyelitis of the frontal bone complicating frontal sinusitis. Laryngoscope 1933; 43: 153-212.

24. Ramadan, H.H.: History of the frontal sinus surgery. Arch Otolaryngol Head Neck Surg 2000; 126: 98-99.

25. Raveh, J., Vuillemin, T., Sutter, F.: Subcranial management of 395 combined frontobasal-midface fractures. Arch Otolaryngol Head Neck Surg 1988; 114: 1114-1122.

26. Raveh, J., Laedrach, K., Speiser, M., et al.: The subcranial approach for fronto-orbital and anteroposterior skull base tumors. Arch Otolaryngol Head Neck Surg 1993; 119: 385-393.

27. Rohrich, R.J., Mickel, T.J.: Frontal sinus obliteration: in search of the ideal autogenous material. Plast Reconstr Surg 1995; 95: 580-585.

28. Schmitz, J.P., Lemke, R.R., Smith, B.R.: The perimeter marking technique for rigid fixation of frontal sinus fractures: Procedure and report of cases. J Oral Maxillofac Surg 1994; 52: $1120-1125$.

29. Shumrick, K.A., Smith, C.P.: The use of cancellous bone for frontal sinus obliteration and reconstruction of frontal bony defects. Arch Otolaryngol Head Neck Surg 1994; 120 : 1003-1009.

30. Tato, J.M., Sibbald, D.W., Bergaglio, O.E.: Surgical treatment of the frontal sinus by the external route. Laryngoscope 1954; 64: 504-521.

31. Wolfe, S.A., Johnson, P.: Frontal sinus injuries: Primary care and management of late complications. Plast Reconstr Surg 1988; 82: 781-791.

Zubillaga-Rodríguez, I.; Falguera-Uceda, M.I.; SánchezAniceto, G.; Montalvo-Moreno, J.J.; Díez-Lobato, R. Abordaje subcraneal. Consideraciones técnicas y aplicaciones en patología traumática craneofacial. Neurocirugía 2010; 21: 467-477.

Correspondencia: Ignacio Zubillaga Rodríguez. Servicio de Cirugía Oral y Maxilofacial. Hospital Universitario 12 de Octubre. Avda. de Córdoba s/n. 28041. Madrid.

e-mail: ignaciozubillaga@yahoo.es 